\title{
Comparaison de la mesure des déformations de fantômes de l'aorte à partir d'image obtenues par IRM et stéréovision
}

\author{
Y. WANG ${ }^{1}$, D. JOANNIC ${ }^{1,2}$, P.DELASSUS ${ }^{3}$, A. LALANDE ${ }^{4}$, P. JUILLION ${ }^{1}$, J.-F. FONTAINE ${ }^{1,2}$ \\ ${ }^{1}$ Laboratoire d'électronique, informatique et image, UMR CNRS 6306, Université Bourgogne Franche-Comté, campus Auxerre, route des \\ plaines de l'Yonne, 89000 AUXERRE, France \\ ${ }^{2}$ IUT Dijon-Auxerre, route des plaine de I'Yonne, 89000 AUXERRE, France \\ ${ }^{3}$ GMedTech, Galway-Mayo Institute of Technology, GALWAY, Ireland \\ ${ }^{4}$ Laboratoire d'électronique, informatique et image, UMR CNRS 6306, Faculté de Médecine, Université Bourgogne Franche-Comté, 7 bd \\ Jeanne d'Arc, 21079 DIJON Cedex, France
}

\begin{abstract}
The study of the wall strain distribution could be helpful to improve the decision criterion for surgery of aortic aneurysm. Recently, numerical simulations can complete the data obtained from imaging measurement in order to develop reliable models. However, the used medical imaging tools are not experimentally validated, in metrological point of view. The aim of this study focused on accuracy and reliability of measurement obtained from kinetic MR sequences. The measures of deformations from MRI were compare to those obtained from stereovision system. Cylindrical phantom of silicone material similar to arterial behavior simulated a symmetric aneurysm was designed. A MRI compatible experimental system reproducing a cardiovascular system with phantom was developed. The acquisition of 40 images per cardiac cycle is triggered with a simulated ECG signal with the same frequency as the pump. In parallel, in-vitro measurement by stereovision system were acquired to obtain local strain by 3D reconstruction with digital image correlation (DIC). The maximum strain calculated with MRI and stereovision at the level of the maximum strain, as the evolution of the strain field along the aneurysm with a pressure of $136 \mathrm{mmHg}$, are close.
\end{abstract}

\section{Introduction}

L'anévrisme est une dilatation permanente et localisée d'une artère avec une augmentation dominante de son diamètre. Il se produit le plus souvent dans l'aorte abdominale. En France, il représente la première cause de décès parmi les maladies cardiovasculaires [1]. Ainsi, la prédiction de la rupture d'un anévrisme de l'aorte abdominale (AAA) est un grand thème d'étude mobilisant de nombreux chercheurs de diverses disciplines telles que la biologie, la mécanique des matériaux, du solide et des fluides, l'imagerie médicale...

Lors de la dernière décennie, de nombreux travaux de modélisation numérique ont été réalisés, en dynamique, avec la prise en compte du couplage fluide-solide (FSI : Fluide Structure Interaction) par la méthode des éléments finis sur des modèles d'anévrismes simples ou réalistes. Parmi ceux-ci, Tezduyar1 et al. [2, 3] ont effectué des simulations d'écoulement sanguin sur des modèles d'artères présentant un anévrisme et ont montré l'existence de phénomène de turbulence, en son sein, ainsi que de leur évolution au cours d'un cycle cardiaque. Un certain nombre de ces travaux ont pour objectif l'étude des contraintes pariétales d'un AAA par diverses modélisations [4 -8]. Il est toutefois notable que ces résultats sont très différents d'un modèle à l'autre compte tenu des hypothèses formulées, elles-mêmes très variables. De ces études, il manque souvent la confrontation et la comparaison à la réalité même si Taylor et Humphrey ont indiqué qu'il y a toujours des écarts entre l'expérimentation et la modélisation numérique [9].

De nombreux développements ont aussi été menés grâce à l'évolution des techniques de mesures. Par exemple, Bihari [10] a réalisé, in vitro, des mesures des déplacements locaux d'un fantôme d'AAA par échocardiographie et mesures par ultrasons. Doyle et al. [11] ont utilisé le procédé de photoélasticimétrie pour étudier les champs de contrainte d'un anévrisme en état statique. Jusqu'à ce jour, très peu de travaux expérimentaux ont été menés sur la mesure, en régime dynamique, de champs de déformation et de vitesse de fantômes déformables. Ces études sont en fait nécessaires afin de mieux appréhender le comportement d'un anévrisme pendant un cycle cardiaque.

L'objet de ce papier est la présentation des résultats d'une étude de faisabilité de mise en œuvre d'un banc d'essai reproduisant le système cardiovasculaire humain en vue de la mesure, par stéréovision, de la déformation d'un fantôme d'anévrisme au cours d'un cycle cardiaque et de la mesure de champs de vitesse d'écoulement sanguin par PIV. Ces résultats sont ensuite confrontés à des résultats obtenus via Imagerie par Résonance Magnétique (IRM) dans des conditions reproductibles des mesures.

\footnotetext{
a Email de correspondance de l'auteur : jffont@iut-dijon.u-bourgogne.fr
} 


\section{Banc expérimental in vitro}

Un banc d'essai reconstituant le système cardiovasculaire a été conçu à partir des composants décrits schématiquement figure 1 . Une pompe à piston, représentant le cœur, délivre 78 pulsations par minute $(1,28 \mathrm{~Hz})$ et assure la propulsion du fluide (dans le cas présent, de l'eau en lieu et place du sang). Des tuyaux assurent l'écoulement du fluide. Leurs raccordements automatiques facilitent l'usage du banc pour différentes configurations environnementales ou lieux de mesure. Les fantômes, modélisant des AAA, sont en silicone et offrent des propriétés matérielles proches de celles des artères. Obtenus par moulage, ils peuvent être de formes complexes et réalistes (cf. figure 2). Une enceinte, en plexiglass, de dimensions $120 \times 60 \times 30 \mathrm{~cm}^{3}$, a été conçue pour immerger les fantômes, dans un liquide de contraste (eau + gadolinium), afin de suivre et mesurer, en IRM, l'évolution de leurs parois interne et externe. Une partie instrumentation assure les réglages du banc et le respect des conditions physiologiques en termes de débit, fréquence et pression. Pour ce faire, nous disposons de deux vannes de régulation de pression, situées en aval de la pompe et en amont du réservoir; de capteurs (PR21SR/80444.3-1 Keller) mesurant la pression en différents endroits du circuit ainsi qu'un débitmètre (Flowmax 22i). La performance du banc a été validée en termes de profil de pression proche de celui du système cardio-vasculaire humain. La régulation du profil de pression est réalisée en agissant sur la course de piston de la pompe ainsi que sur les vannes. Cette forme générale de profil est conservée tout au long d'un cycle de mesure.

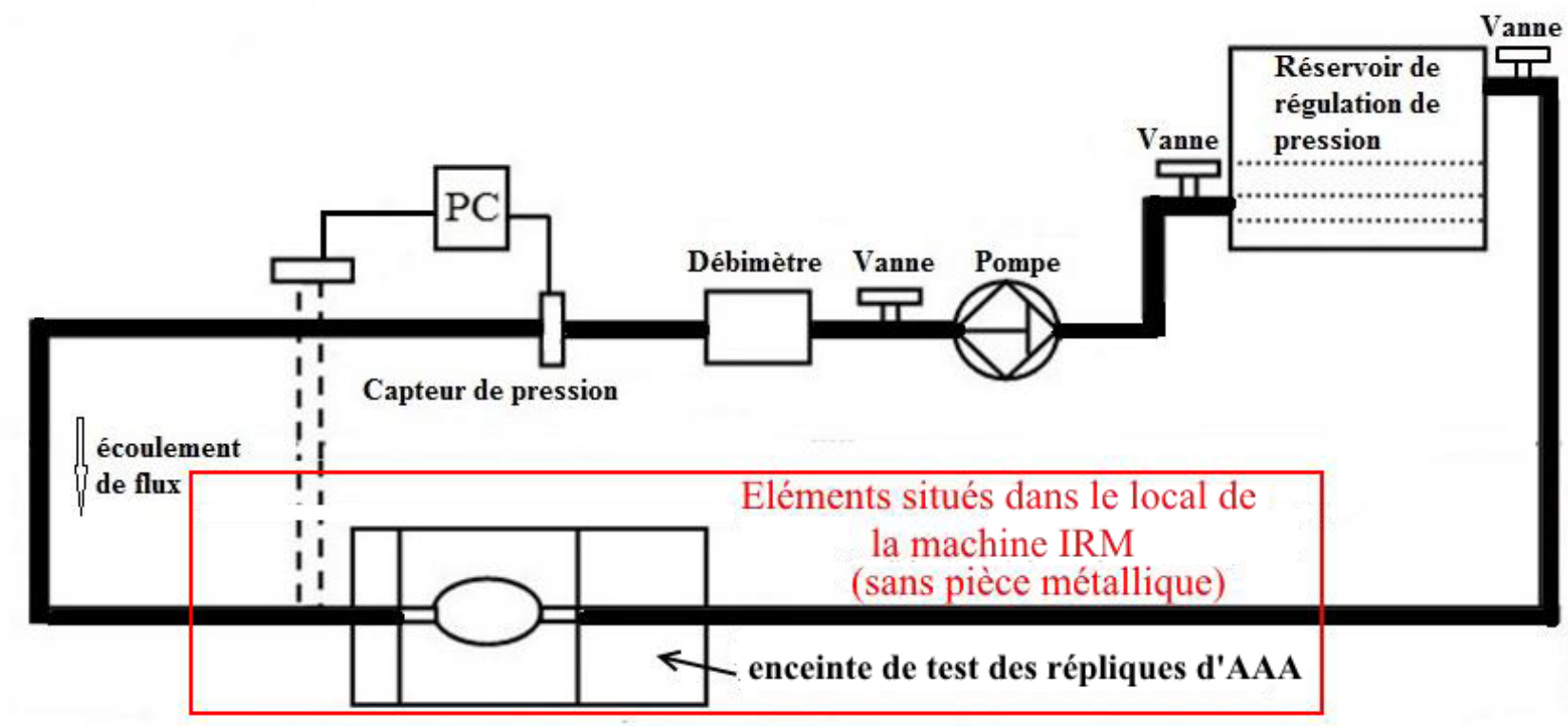

Figure 1. Dispositif expérimental.
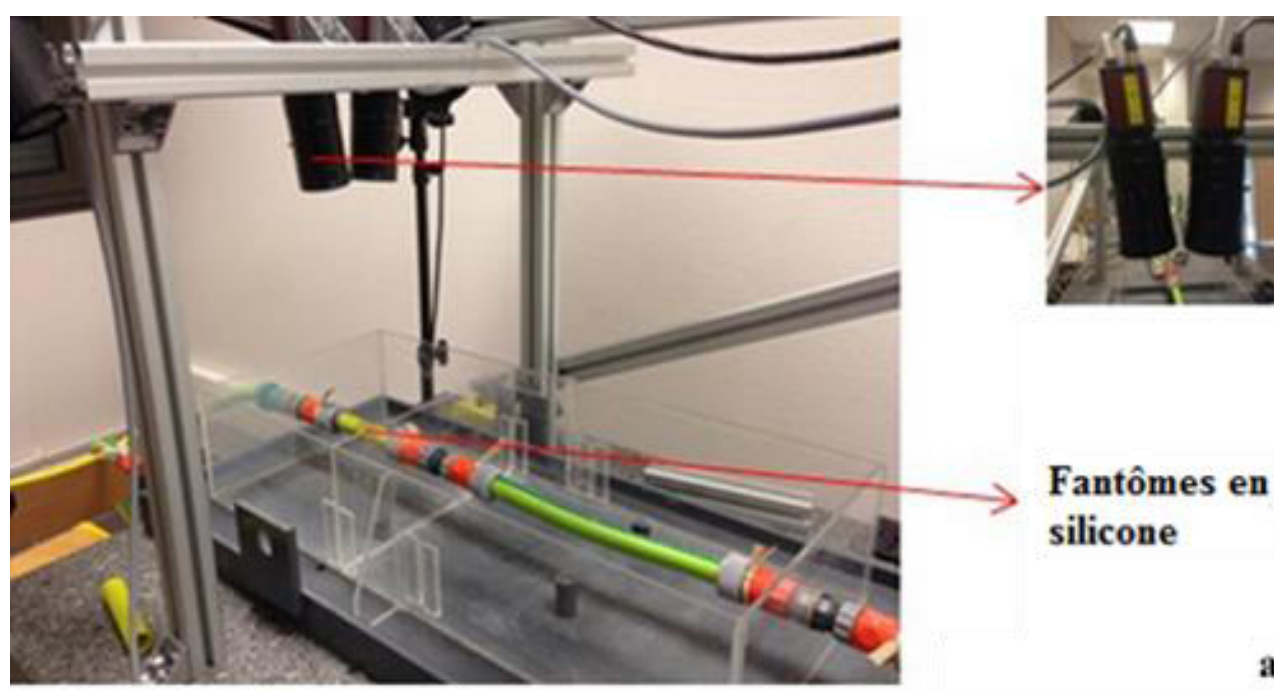

\section{Caméras et objectifs}

\section{Fantômes en silicone}
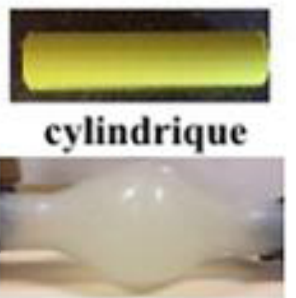

avec AAA axisymétrique

Figure 2. Dispositif de mesure par stéréovision et enceinte de test des fantômes 


\section{Dispositif de stéréovision}

\subsection{Processus de mesure}

Le dispositif de stéréovision est visible sur la figure 2. Le processus de mesure par stéréovision mis en œuvre est décrit figure 3.

En premier lieu et en vue de la reconstruction $3 \mathrm{D}$, le calibrage des caméras (1) est effectué selon la méthode développée par Faugéras et Toscani [13]. La mesure de champs de déformation étant obtenue par la technique de correction d'images (DIC), la peau des fantômes est rendue texturée, soit au moulage par l'ajout de particules dans le silicone, soit à l'issue du moulage, par l'application de fines particules de peinture créant un mouchetis au motif aléatoire (2). Le dispositif de stéréovision (3) est constitué de deux caméras Manta MJ504B de la société Allied Vision Technologies. Fixées sur un support rigide, elles sont réglables en position et en orientation (voir figure 2). Les objectifs des caméras et leurs réglages optiques étant identiques, les biais dus aux différences de résolution, de luminosité et de contraste des images sont évités. La synchronisation des prises de vues est réalisée avec un trigger externe Arduino ${ }^{\circledR}$. Le logiciel $7 \mathrm{D} \AA$, développé par le laboratoire SYMME de l'université de Savoie, est utilisé pour réaliser l'analyse d'images. Il permet d'obtenir une cartographie des déplacements et des déformations de Green-Lagrange entre deux configurations à partir de la technique de corrélation d'images (4).

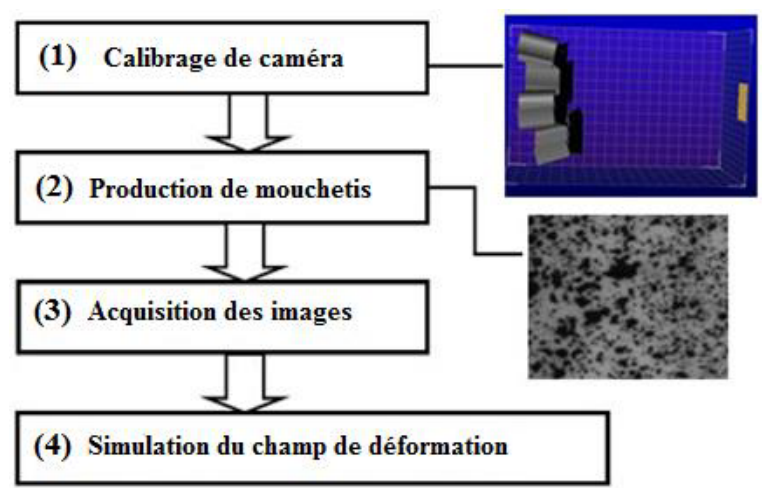

Figure 3. Processus de mesure par stéréovision et DIC

\subsection{Cadence de mesure et résolution}

La maîtrise de la qualité de la mesure, et par conséquent du banc expérimental, nécessite le réglage des caméras en termes de résolution de l'image et de fréquence d'acquisition et ce, pour des conditions de flux en mode dynamique. En effet, le choix de la cadence de mesure est déterminé de manière à avoir un nombre d'images par seconde le plus élevé possible avec un bruit de mesure minimal. En théorie, les caméras peuvent prendre jusqu'à 9 images par seconde pour une résolution complète de $2448 \times 2056$ pixels et 15 images par seconde pour une résolution réduite de $1224 \times 1028$ pixels.
Ainsi, l'influence de la résolution sur le résultat de mesure d'un déplacement a été testée sur un échantillon plan texturé à l'aide d'une table micrométrique étalonnée. Un déplacement $u_{0}$ est imposé par une table micrométrique. Des images ont été acquises puis traitées pour différentes résolutions. Le tableau 1 en donne des résultats de mesure de déplacement $u$ pour deux niveaux de résolution.

Tableau 1. Mesure de déplacement imposé $u_{0}$ pour deux niveaux de résolution

\begin{tabular}{c|cc}
\hline \multirow{2}{*}{$u_{0}=4.5$} & \multicolumn{2}{|c}{ Résolution des images (pixels) } \\
\cline { 2 - 3 } $\mathrm{mm}$ & $1224 \times 1028$ & $2448 \times 2056$ \\
\hline$u(\mathrm{~mm})$ & 4.65 & 4.49 \\
\hline$e(\mathrm{~mm})$ & 0.15 & 0.01 \\
\hline$\sigma(\mathrm{mm})$ & 0.016 & 0.0014 \\
\hline
\end{tabular}

Ces résultats ont été corrélés pour d'autres niveaux de résolution. On remarque que plus la résolution est élevée, plus faibles sont les erreurs et les dispersions de mesure. Des essais ont également été menés sur un fantôme cylindrique mis sous pression hydrostatique pour différentes résolutions. Dans le cas d'images à haute résolution, les résultats sont satisfaisants en termes d'homogénéité du champ de déformation. Pour la plus basse résolution, la dispersion $\sigma$ indique un champ de déformation non homogène (voir tableau 2). On note un faible écart sur le niveau de déformation $\varepsilon$ entre les résultats expérimentaux pour une haute résolution et les résultats numériques. Il provient en fait du bruit de mesure.

En conclusion de ces essais, nous retenons que le choix de la résolution complète, ie $2448 \times 2056$ pixels, est la meilleure. Ce choix limite toutefois à une fréquence d'acquisition à 9 images par seconde.

Tableau 2. Résultats de mesures de déformation et dispersion associée sur fantôme cylindrique soumis à pression hydrostatique

\begin{tabular}{c|cc}
\hline \multirow{2}{*}{$\begin{array}{c}\mathrm{p}=232 \\
\text { mbar }\end{array}$} & \multicolumn{2}{|c}{ Résolution des images } \\
\cline { 2 - 3 } & $1224 \times 1028$ & $2448 \times 2056$ \\
\hline$\varepsilon(\%)$ & 18.2 & 16 \\
\hline$\sigma(\%)$ & 19.8 & 3.4 \\
\hline
\end{tabular}

Par la suite, une série d'expériences a été entreprise sur le fantôme cylindrique sous pression dynamique afin de tester l'influence de différentes cadences d'acquisition sur la mesure des déformations. La fréquence de battement du fantôme, simulant le rythme cardiaque, est de $1,26 \mathrm{~Hz}$ et le débit moyen est de 4 litres/min. Les mesures de déformations ont été effectuées sur la partie centrale du fantôme (pour éviter les effets de bord). Les résultats de ces essais sont donnés dans le tableau 3. Pour une cadence supérieure à 8 images par seconde, on note une distribution non homogène du champ de déformation. Insatisfaisants, ces résultats s'expliquent par l'aspect dynamique de l'essai, et la difficulté de synchroniser les deux caméras avec le mouvement subi par le fantôme. 
Pour une cadence inférieure ou égale à 7 images par seconde, le champ de déformations est homogène quelle que soit la cadence retenue.

Tableau 3. Déformation $\varepsilon$ vs cadence d'acquisition (cas d'un fantôme cylindrique sous pression dynamique)

\begin{tabular}{ccc}
\hline $\begin{array}{c}\text { Cadence } \\
\text { (image/s) }\end{array}$ & $\boldsymbol{E}_{\text {MAX }}$ & Distribution \\
\hline $\mathbf{3}$ & $16.01 \%$ & Homogène \\
\hline $\mathbf{4}$ & $16.05 \%$ & Homogène \\
\hline $\mathbf{5}$ & $16.06 \%$ & Homogène \\
\hline $\mathbf{6}$ & $16.11 \%$ & Homogène \\
\hline $\mathbf{7}$ & $16.14 \%$ & Homogène \\
\hline $\mathbf{8}$ & - & Non homogène \\
\hline $\mathbf{9}$ & - & Non homogène \\
\hline
\end{tabular}

Pour affiner cette recherche de cadence optimale de mesure, le suivi de la déformation du fantôme a été étudié au cours du temps et sur un cycle de 0.79 s. Pour se placer dans le cadre de la comparaison des mesures à celles de l'IRM (40 images prises lors d'un cycle cardiaque), il est nécessaire d'effectuer l'acquisition en stéréovision sur plusieurs cycles ; cette mesure étant équivalente puisque le dispositif mis en œuvre (pompes, comportement des tubes,...) est suffisamment stable et n'entraîne pas de variation dans le temps. Après acquisition, une mise en correspondance temporelle des images est réalisée sur un seul cycle.

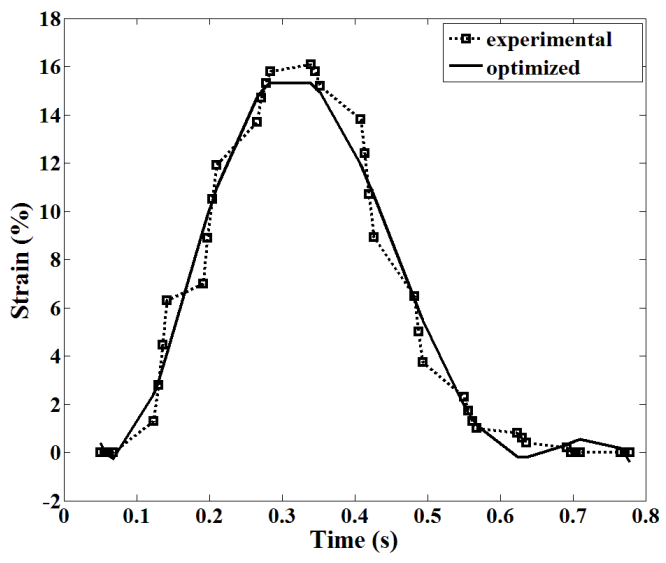

Les figures 4 représentent les courbes reconstruites de déformation $\mathrm{du}$ fantôme au cours d'un cycle respectivement pour les cadences de 3 et 7 images par seconde. Sur chacune de ces figures, une courbe est obtenue par optimisation polynomiale avec la méthode des moindres carrés.

Pour chaque cadence de mesure, on note des fluctuations assez importantes des points de mesures autour de la courbe optimisée. Afin de déterminer la fréquence d'acquisition idéale, nous avons appliqué une fonction de somme des écarts à chacun de ces essais. La fonction est donnée équation (1) :

$$
\xi=\sum_{i=1}^{40}\left|\varepsilon_{i}-y\left(t_{i}\right)\right|
$$

avec $\varepsilon_{i}$, la déformation, et $y\left(t_{i}\right)$ la fonction polynomiale optimisée. Les résultats sont donnés dans le tableau 4 : la cadence optimale de mesure est de 7 images par seconde. Cette valeur est retenue pour la suite des travaux.

Tableau 4. Résultats, par cadence, de la fonction somme des écarts

\begin{tabular}{cccccc}
\hline $\begin{array}{c}\text { Nb. } \\
\text { Images/s }\end{array}$ & 3 & 4 & 5 & 6 & 7 \\
\hline$\xi$ & 86.5 & 77.6 & 59.9 & 53.0 & 30.8 \\
& 5 & 8 & 2 & 7 & 1 \\
\hline
\end{tabular}

Figure 4. Courbes de déformation vs temps pour une cadence de 3 images/s (à gauche) et de 7 images/s (à droit)

\section{Comparaison des résultats de mesure par IRM et par stéréovision}

Les essais et acquisitions (par stéréovision et IRM) ont été réalisés dans des conditions semblables à l'aide d'un fantôme d'AAA de forme axisymétrique, cf. figure 2. Les paramètres du banc étaient les suivants : fréquence de

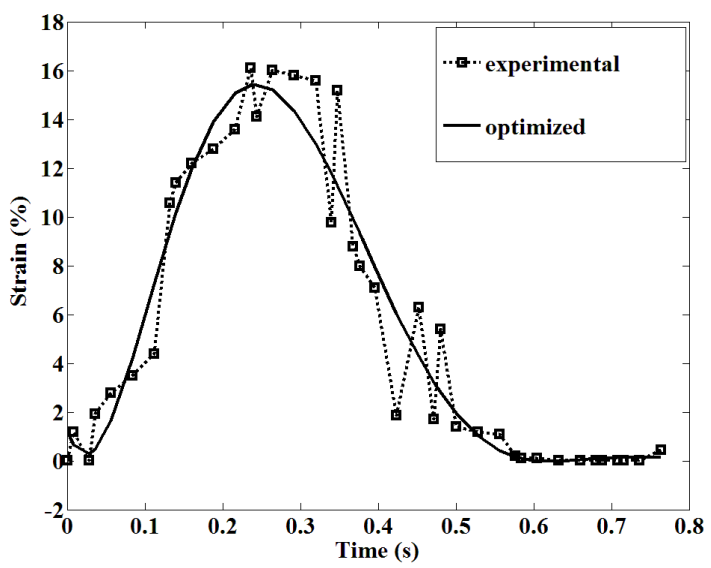

flux cardiaque de 1,26 Hz, débit moyen de $4 \mathrm{~L} / \mathrm{min}$ et pression relevée à l'entrée du fantôme de 124 mbar. En ce qui concerne l'acquisition par IRM, 40 séquences d'acquisition de 22 coupes transversales du fantôme ont été réalisées selon l'axe longitudinal pendant la durée d'un cycle. 

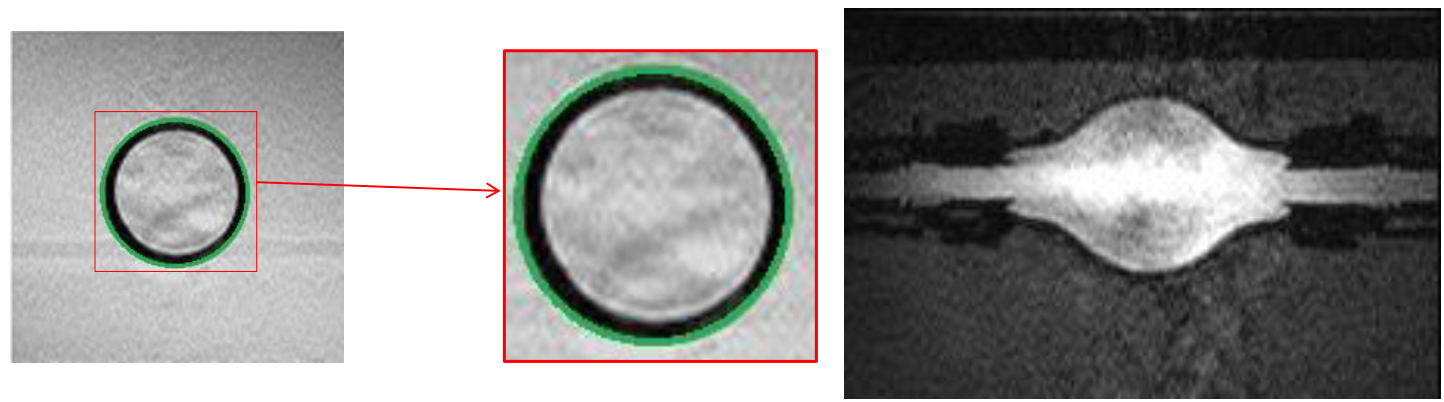

Figure 6. Exemple d'image d'un fantôme avec AAA acquise par IRM en séquence cinétique : coupe transversale (à gauche) et coupe longitudinale (à droite)

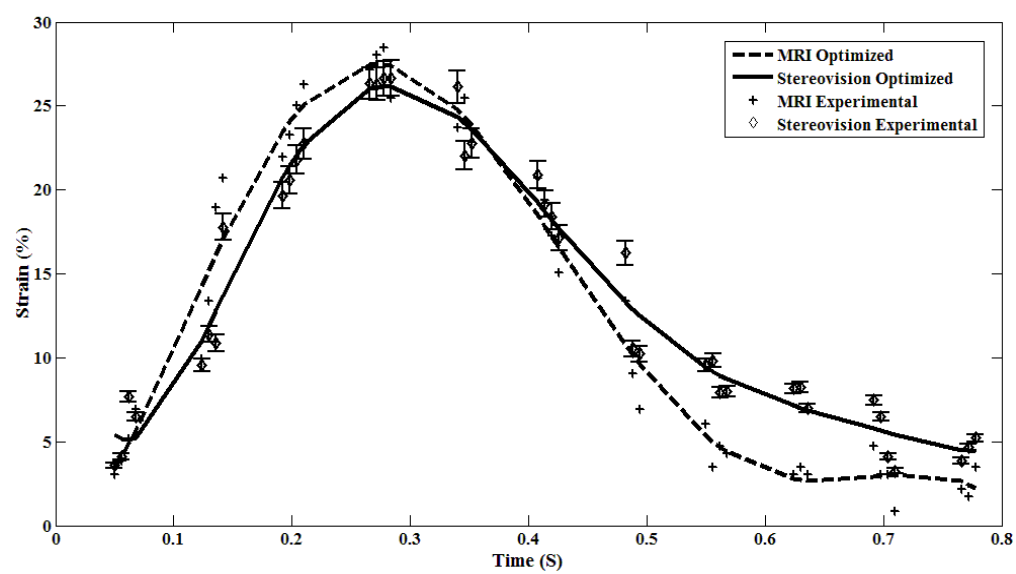

pression

(mbar)

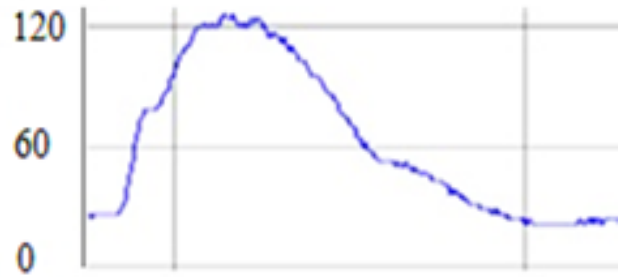

0,2 temps (s) $\quad 0,7$

Figure 7. Comparaison de profils de déformations maxi d'un fantôme avec anévrisme axisymétrique, déduites des techniques d'imagerie par IRM et stéréovision (à gauche) suivant le cycle de pression (à droite)

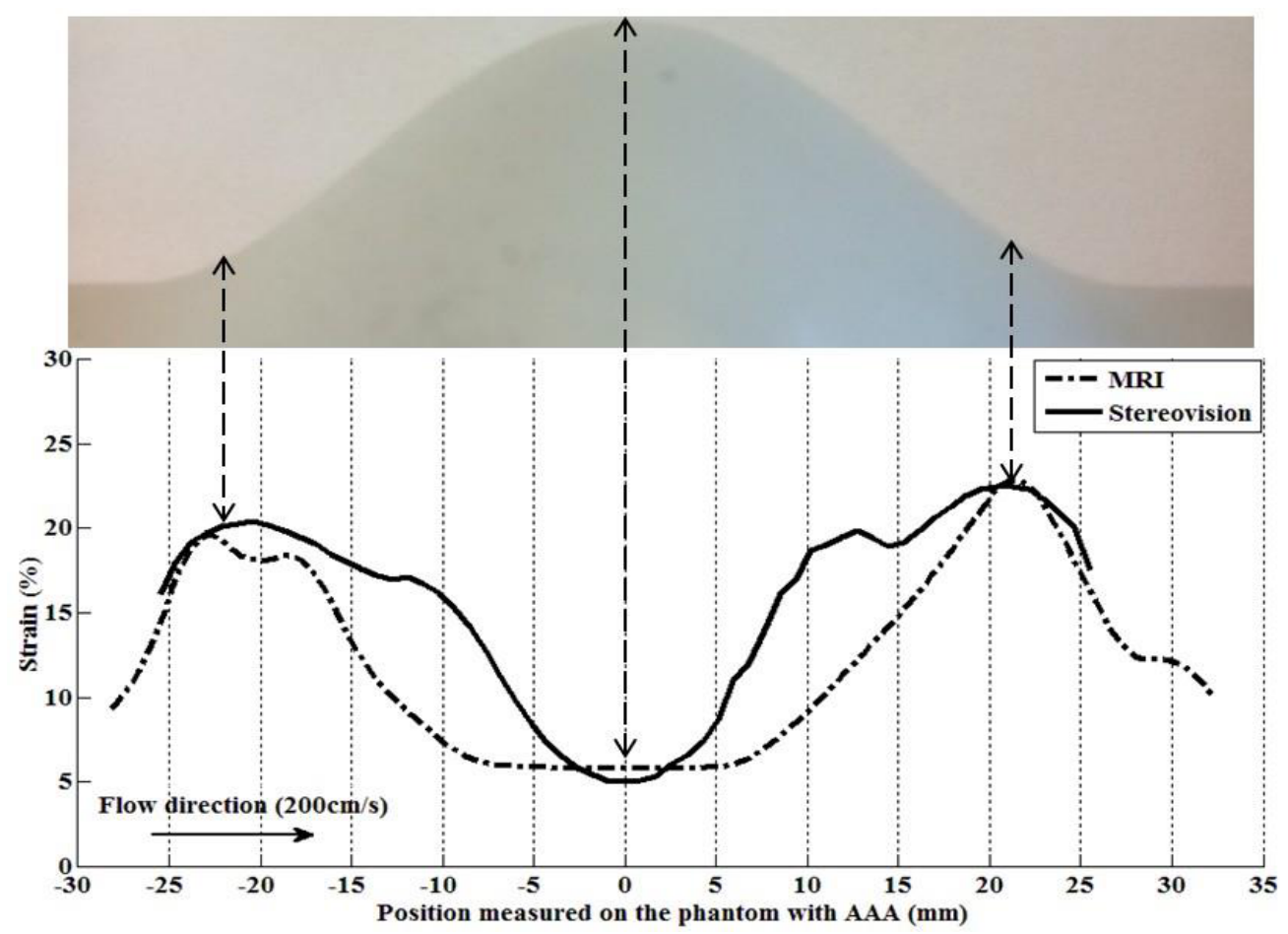

Figure 8. Comparaison de la répartition selon l'axe longitudinal à la pression maxi des déformations d'un fantôme avec anévrisme axisymétrique, déduites des techniques d'imagerie par IRM et stéréovision 
Pour chaque coupe transversale, il est d'alors possible de remonter à l'information de déformation $\varepsilon$ par la mesure de la variation relative de diamètre du fantôme. La figure 6 montre un exemple d'images obtenues par IRM en séquence cinétique correspondant à deux états du cycle. La figure 7 compare des profils de déformation, relevés selon l'axe l'anévrisme, et obtenus respectivement par stéréovision et IRM. Il est notable que les courbes d'évolution sur le cycle ont des profils relativement similaires. Les deux courbes de répartition axiales données sur la figure 8 ne sont pas tout à fait corrélées, une des raisons envisagées est la différence d'échantillonnage entre les essais. En IRM, seules 22 coupes transversales du fantôme ont été acquises contre 62 en stéréovision. D'autre part, ces deux techniques de mesure utilisent deux méthodes d'évaluation des déformations différentes. Par la technique de corrélation d'images, des mesures de déformations locales sont effectuées tandis que du traitement des images acquises par IRM une déformation globale est estimée.

\section{Conclusion}

Un banc expérimental d'étude des systèmes cardiovasculaires, plus précisément de l'aorte, a été élaboré avec une problématique de reproductibilité des mesures dans une ambiance contrainte (sans présence de métal). Des expérimentations ont été effectuées sur des fantômes cylindriques caractérisant une artère saine mais aussi sur des fantômes présentant un anévrisme axisymétrique. Les résultats obtenus montrent que les mesures par IRM sont bien corrélées aux mesures effectuées par stéréo-corrélation d'images, technique qui est bien adaptée à la mesure 3D des déformations en surface. Cette première étude, effectuée dans un cadre métrologique, va être approfondie afin de caractériser la capacité de l'IRM à mesurer des déformations sur des fantômes de formes complexes plus réalistes mais aussi des vitesses de fluide qui sont nécessaires pour développer un outil d'aide au diagnostic des pathologies de type anévrisme de l'aorte.

\section{Références}

1 V. Lacroix, I. Aboyans, A. Guessous, P. Leclerc, De la recommandation d'un dépistage à son (non) implantation : le cas de l'anévrisme de l'aorte abdominale, Revue Médicale Suisse N ${ }^{\circ} 3256$, (2009).

2 T.E Tezduyar1, S. Sathe, T. Cragin, B. Nanna, B.S. Conklin, J. Pausewang, M.Schwaab, "Modelling of fluid-structure interactions with the space-time finite elements: Arterial fluid mechanics", Int. J. Num. Meth. Fluids, 54, 901-922, (2007).

3 T.E.Tezduyar1, S.Sathe1, M. Schwaab, B.S., Conklin, Arterial fluid mechanics modeling with the stabilized space-time fluid-structure interaction technique, Int. J. Num.. Meth. Fluids,: 57, 601-629. (2008).

4 B.J.B.M Wolter., M.C.M. Rutten, G.W.H. Schurink, U. Kose, J.de Hart, F.N. Van de Vosse, A patient- specific computational model of fluid-structure interaction in abdominal aortic aneurysms, Medical Engineering \& Physics , 27, 871-883 (2005).

5 P. Rissland, Y. Alemu, S. Einav, J. Ricotta, D. Bluestein, Abdominal aortic aneurysm risk of rupture: patient-specific FSI simulations using anisotropic model, J. of Biomechanical Engineering 131, 03, 1001 (2009).

6 C.M. Scotti, E.A. Finol, Compliant biomechanics of abdominal aortic aneurysms: A fluid-structure interaction study, Computers \& Structures, 85, 1114: 1097-1113. (2007).

7 C.M. Scotti, J.Jimenez, S.C. Muluk, E.A. Finol, Wall stress and flow dynamics in abdominal aortic aneurysms: finite element analysis vs fluid-structure interaction, Computer Methods in Biomechanics and Biomedical Engineering, 11, 301-322. (2008).

8 ZY Li, J U-King-Im, TY Tang, E Soh, TC See, JH Gillard, Impact of calcification and intraluminal thrombus on the computed wall stresses of abdominal aortic aneurysm, J. of Vascular Surgery: 47, 928-935 (2008).

9 C.A. Taylor, J.D. Humphrey, Open problems in computational vascular biomechanics: Hemodynamics and arterial wall mechanics , Comput. Methods Appl. Mech. Eng., 198, 45-46, 3514-3523 (2009).

10 P.Bihari, A. Shelke, T.H. Nwe, M. Mularczyk, K. Nelson, T. Schmandra, P. Knez, T. Schmitz-Rixen, Strain Measurement of Abdominal Aortic Aneurysm with Real-time 3D Ultrasound Speckle Tracking, European Journal of Vascular and Endovascular Surgery, 45 04, 315-323. (2013).

11 B.J. Doyle, J. Killion, A. Callanan, Use of the photoelastic method and finite element analysis in the assessment of wall strain in abdominal aortic aneurysm models, J. of Biomechanics, 45, 10, 1759 1768 (2012).

12 Faugéras, O.D., Toscani, G., 1987. Camera calibration for 3-D computer vision, in Proceedings of IEEE International Workshop on Machine Vision and Machine Intelligence, Tokyo, Japan.

\section{Remerciements}

Les auteurs remercient le conseil régional de Bourgogne qui par l'intermédiaire des fonds FEDER a contribué au financement de cette étude. 